\title{
Lymphangiography: A Case Study
}

\author{
Labib H. Syed, M.D., M.P.H., ${ }^{1}$ Christos S. Georgiades, M.D., Ph.D., ${ }^{1}$ \\ and Vicki L. Hart, R.T.(R)
}

ABSTRACT

Lymphatic leak is a rare but well-described complication of a multitude of surgeries, whose sequela may potentially be life threatening. For cases refractory to conservative management, surgical therapy has been the mainstay of treatment. Although radiology has always played a contributory role in the diagnosis of lymphatic leaks with lymphoscintigraphy and lymphangiography, minimally invasive management of lymphatic leaks by interventional radiologists has only been described in the last decade. We present a case of percutaneous disruption of the cisterna chyli to treat a lymphatic leak of the thoracic duct.

KEYWORDS: Lymphangiogram, lymphatic leak, cisterna chili

\begin{abstract}
Objectives: Upon completion of this article, the reader should be able to discuss the role of a lymphangiogram and percutaneous embolization or disruption techniques in the treatment of lymphatic leaks.

Accreditation: Tufts University School of Medicine (TUSM) is accredited by the Accreditation Council for Continuing Medical Education to provide continuing medical education for physicians.

Credit: TUSM designates this educational activity for a maximum of 1 AMA PRA Category 1 Credit $^{\mathrm{TM}}$. Physicians should only claim credit commensurate with the extent of their participation in the activity.
\end{abstract}

Lymphatic leak is a rare but well-described complication of a multitude of surgeries. These include radical neck dissections, coronary artery revascularization with left or right internal mammary artery grafts, retroperitoneal lymph node resections, and peripheral vascular surgical procedures. Even within the realm of interventional radiology, lymphatic leaks have been described as a rare complication of central venous catheter insertion. ${ }^{1}$ Other etiologies of leaks include trauma and as a complication of radiation therapy in cancer patients. ${ }^{2}$ The sequelae of such leaks may potentially be life threatening secondary to the loss of volume, plasma proteins, triglycerides, and immunoregulatory lymphocytes. The combined effect of these losses results in an immunocompromised state in which individuals are highly susceptible to infections.
Treatment has traditionally been conservative, with diet modification to include a high-protein, low-fat, medium-chain triglyceride diet with total parenteral nutrition. ${ }^{3,4}$ Therapeutic paracentesis and chest tube placement for chylous ascites and chylothorax, respectively, provide symptomatic relief. For cases refractory to conservative management, thoracotomy with surgical ligation of the thoracic duct has been the mainstay of treatment, at least for thoracic leaks. Advances in surgical technique also permit a minimally invasive approach utilizing video-assisted thoracic surgery (VATS) in the ligation of the thoracic duct. Radiology has always played a contributory role in the diagnosis of lymphatic leaks with lymphoscintigraphy and lymphangiography, the latter of which may also be therapeutic. ${ }^{5}$ Interventional radiology first arrived in the
${ }^{1}$ Vascular and Interventional Radiology, Johns Hopkins Hospital, Baltimore, Maryland; ${ }^{2}$ Department of Imaging Sciences, University of Rochester, Rochester, New York.

Address for correspondence and reprint requests: Labib H. Syed, M.D., M.P.H., Chief, Clinical Fellow, Vascular and Interventional Radiology, Johns Hopkins Hospital, 600 N. Wolfe Street, Blalock 545, CVDL, Baltimore, MD 21287.
Case-Based Interventional Radiology; Guest Editors, Ron C. Gaba, M.D., Derek L. West, M.D., James T. Bui, M.D., Charles A. Owens, M.D.

Semin Intervent Radiol 2007;24:106-110. Copyright (C) 2007 by Thieme Medical Publishers, Inc., 333 Seventh Avenue, New York, NY 10001, USA. Tel: +1(212) 584-4662.

DOI 10.1055/s-2007-971180. ISSN 0739-9529. 
treatment of lymphatic leaks in Cope's landmark article in $1998 .{ }^{6}$ He described a technique involving the performance of a lymphangiogram to obtain percutaneous access to the cisterna chyli. From the cisterna chyli, the thoracic duct was opacified and subsequently embolized. We present a case of percutaneous disruption of the cisterna chyli to treat a lymphatic leak of the thoracic duct.

\section{CASE REPORT}

We present the case of a 70-year-old white man with a $5.5-\mathrm{cm}$ saccular aneurysm of the thoracic aorta amenable for endovascular repair, which was incidentally discovered on a computed tomography (CT) scan. Repair of the aneurysm was complicated by occlusion of one of the carotid arteries, and consequently a staged procedure was planned with a jump graft from one carotid artery to the other in preparation for the endovascular repair in the future. The patient's past medical history was significant for type 2 diabetes, hypertension, chronic obstructive pulmonary disease, and a 40 pack-year history of smoking.

The patient underwent the first stage of his procedure with a right to left carotid artery bypass using an $8-\mathrm{mm}$ polytetrafluoroethylene (PTFE) graft. The surgery was uneventful, but due to the extensive retropharyngeal dissection, the patient failed two extubations secondary to pharyngeal edema. The patient was left intubated in the intensive care unit for a week until the edema resolved. The patient was finally extubated and transferred to the floor, where an oral diet was started. On the second day after initiation of the diet, the patient developed a low-output chyle leak. This was managed conservatively with lipid-free total parenteral nutrition (TPN) and $100 \mu \mathrm{g}$ of octreotide, subcutaneously administered every 8 hours. Over the next few days, the leak worsened to 1 to $3 \mathrm{~L}$ of chyle per day, and a wound vacuum was placed on the wound. At this time, interventional radiology was consulted for a lymphangiogram.

\section{LYMPHANGIOGRAM TECHNIQUE AND APPLICATION IN OUR PATIENT}

Prior to the procedure, a standard interventional radiology workup is performed. A careful history of current medications is reviewed, with particular attention to blood-thinning agents. Acceptable coagulation parameters include an international normalized ratio $\leq 1.8$ and a platelet count of at least 50,000. If abnormal, these values may be corrected with transfusion of fresh-frozen plasma or platelets. A complete and thorough surgical history is obtained because this is the most likely culprit. Prior radiation exposure or a travel history with exposure to filariasis are much less likely causes of a lymphatic leak or obstruction. Once these criteria are met, informed consent is obtained outlining the risks, benefits, and alternatives to the procedure. The main risks associated with the procedure are infection and pain, although they are exceedingly rare. Alternatives to this procedure involve open surgery. Mere performance of a lymphangiogram abates a lymphatic leak in 50 to $75 \%$ of cases because the opacifying agent (lipiodol) is also a sclerosing agent. If the leak involves the thoracic duct, direct embolization of the cisterna chyli may be performed to improve success rates. If lymphatic obstruction is suspected or only localization of a leak is desired, a lymphoscintigram performed with a radioisotope may be useful.

In patients with suspected lymphatic leak who are stable, steps may be taken to exacerbate the leak before the procedure to increase sensitivity of the lymphangiogram. These steps may include halting of TPN and commencement of a high-fat diet. Patients who present with chylothorax or chylous ascites should also be drained prior to the lymphangiogram to further provoke the leak.

The patient is brought into the interventional radiology suite and placed supine on the table. Both feet are positioned flat on the table by placement of a triangular wedge under the knees resulting in slight plantar flexion. The feet are then prepared and draped so that both dorsums are exposed. A 1-mL mixture

A
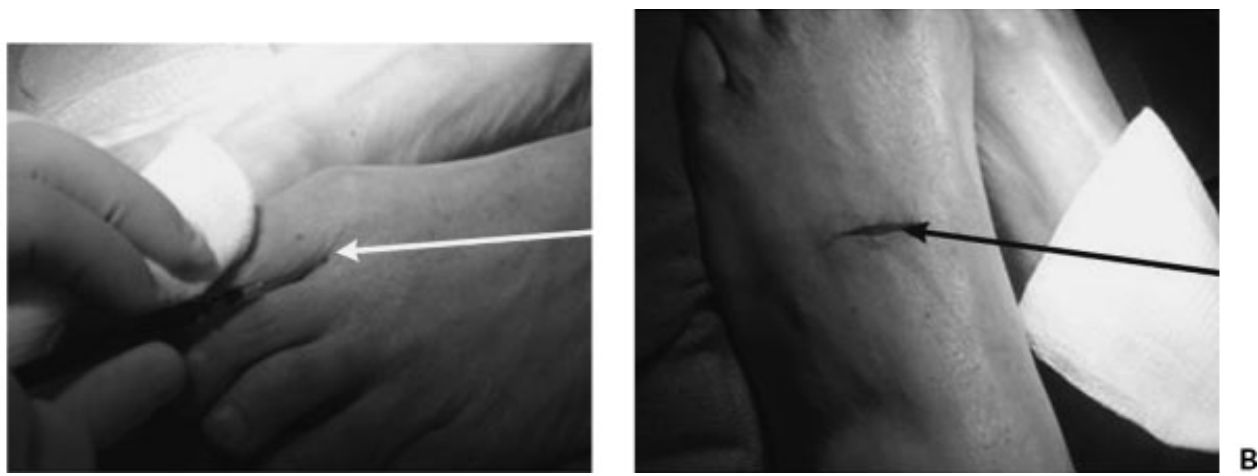

Figure 1 (A) Photograph of foot during subcutaneous injection of $75 \%$ methylene blue and $25 \%$ epinephrine into web space between digits 1 and 2 with 22-gauge needle (white arrow). (B) Photograph of feet following superficial incision along dorsum of foot to dissect out lymphatics (black arrow). 
consisting of $75 \%$ methylene blue and $25 \%$ epinephrine is injected subcutaneously with a 22-gauge needle into the web spaces between digits 1 and 2, 2 and 3 , and 3 and 4 bilaterally (Fig. 1). The blue-stained lymphatics are identified over the dorsum of the foot, and after infiltration of the overlying skin with lidocaine and epinephrine on the dorsum of each foot, a $2-\mathrm{cm}$ horizontally oriented superficial incision is made with a scalpel. The lymphatics are bluntly dissected out using small scissors and forceps. Slow careful dissection is necessary to avoid injury to vessels, which may obscure the field as methylene blue or blood floods the area. A small piece of triangular-shaped paper is placed under the lymphatic vessels to elevate them and make them more visible. Silk ties are then placed under the lymphatics in proximal and distal positions. The lymphatic vessel is then accessed using a 30-gauge lymphangiogram needle. Use of magnifying glasses are necessary for this step of the procedure. A very gentle saline injection using a 1-mL syringe confirms positioning within the lymphatic vessel, and the distal silk tie is

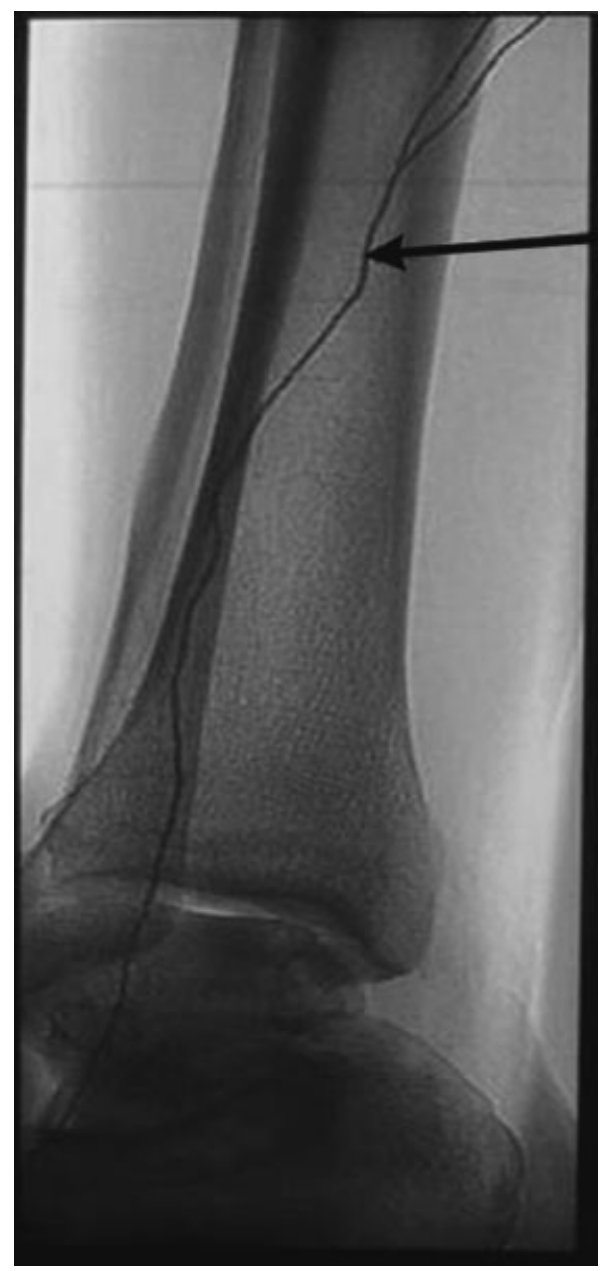

Figure 2 Lateral radiograph of ankle demonstrates Ethiodol tracking uniformly through the lymphatic system (arrow). In contrast, due to its oil base, Ethiodol forms globules in the vascular system, thereby differentiating the two. looped and tied at the level just proximal to the needle bevel. This secures the tip of the needle in the lymphatic vessel. The catheter portion of the needle is then secured to the skin. The needle is then connected to a power injector, which deploys syringes of heated lipiodol at a rate of $7 \mathrm{~mL}$ per hour. Moistened pads are placed over the lymphatics to prevent dehydration. Intermittently, spot radiographs of the opacified lymphatics are taken in a progressively cranial manner, the first one to ensure access into the lymphatics over the foot (Fig. 2). It may take a few minutes or a couple of hours for the lipiodol contrast to reach the abdomen, so regular fluoroscopic observation is necessary (Figs. 3 and 4).

Once the appropriate lymphatics are opacified and a leak (or obstruction) is demonstrated, the next step is to decide whether the patient can benefit from intervention. If the leak is after surgery and at the level of the thoracic duct, lymphatic embolization may be beneficial to the patient.

With the cisterna chyli opacified, limited abdominal ultrasound was performed in our patient to show the cisterna chyli. The positions of the aorta and inferior vena cava were noted. An appropriate skin access site and trajectory was chosen, and the area was prepared and draped in the usual sterile fashion. Lidocaine was administered for local pain control. Utilizing triangulation techniques and ultrasound guidance, a 19-gauge needle

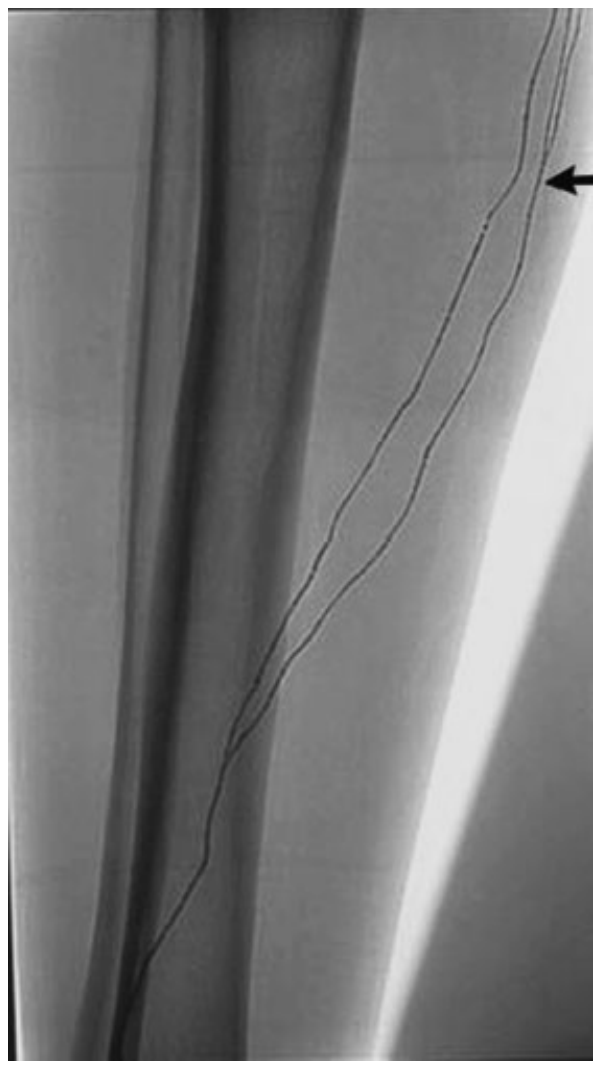

Figure 3 Intermittent spot radiograph of calf reveals opacified lymphatics (arrow) with cranial progression of Ethiodol. 


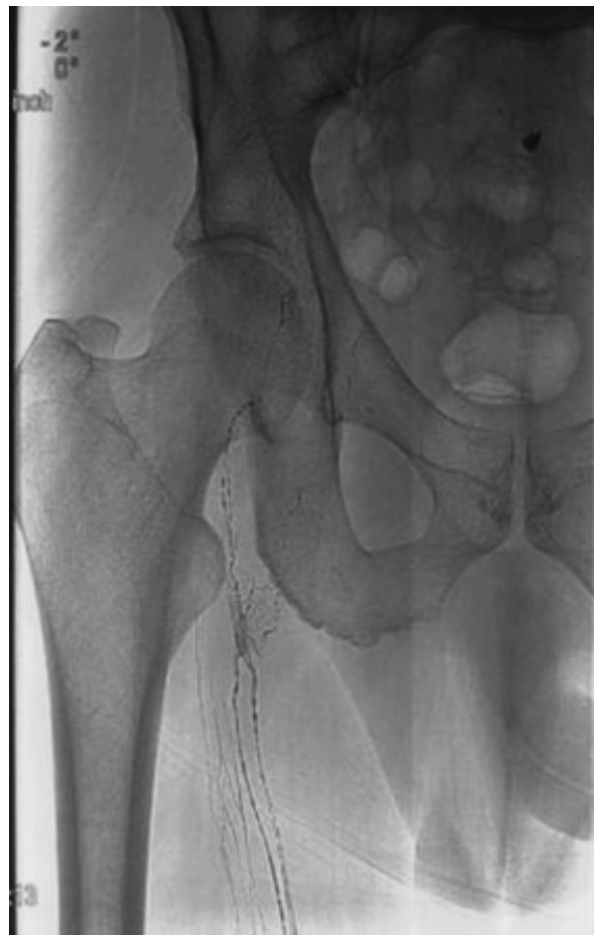

Figure 4 Radiograph of right upper thigh reveals cranial progression of Ethiodol.

was advanced into the cisterna chyli. An attempt was made to pass a 0.018 Microvena wire (Microvena, Whitebear, MN) into the thoracic duct without success. We then injected $1 \mathrm{~mL}$ of an alcohol and lipiodol mixture in a 1:1 ratio to sclerose the cisterna chyli. Images of the disrupted cisterna chyli were taken (Fig. 5). The needle was then removed and a skin dressing applied. The skin overlying the dorsum of both feet was irrigated and closed with subcuticular suture. Ideally, the objective is to embolize the thoracic duct with coils or glue. However, disruption of the cisterna chili may have the same effect.

Our patient was followed up after the procedure. Within 1 week the lymphatic drainage at the left neck and chest wound site gradually decreased until it finally resolved completely.

\section{DISCUSSION}

Lymphatic leak is a serious but fairly uncommon complication of surgery, trauma, or radiation treatment. Treatment is initially conservative and includes diet modification such as lipid-free TPN, drainage with chest tubes or paracentesis, and pharmacological agents such as octreotide. If these measures are unable to control the leak, surgical intervention has been the standard of care. However, many individuals with lymphatic leaks are poor surgical candidates, and oftentimes the location of the leak is difficult to ascertain. Consequently, a lymphoscintigram may be used to

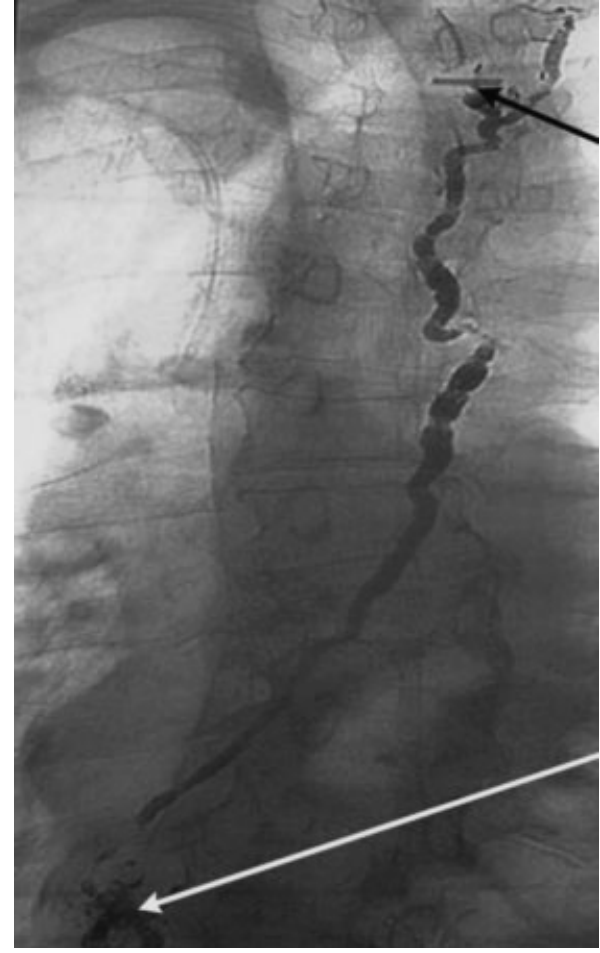

Figure 5 Chest radiograph reveals Ethiodol opacifying thoracic duct. Thoracic duct travels cranial and anterior to drain into subclavian vein. Black arrow points to surgical clip and site of leak. Ethiodol is left within the thoracic duct to promote sclerosis. Cisterna chyli has lost its normal globular appearance and is disrupted after ethanol ablation (white arrow).

identify a leak or obstruction and a lymphangiogram may be used as both a diagnostic and therapeutic test. In conjunction with a lymphangiogram, direct percutaneous embolization of the thoracic duct or disruption of the cisterna chyli may be performed as definitive treatment, as in this case.

Among surgical categories, radical neck dissections and esophageal surgeries are most often complicated by lymphatic leaks. These have been treated traditionally by surgical ligation of the thoracic duct. Unfortunately, this procedure may have mortality and morbidity rates as high as $25 \%$ and $39 \%$, respectively. ${ }^{7,8}$ By contrast, a simple lymphangiogram has reported cases of mortality and morbidity rates of $<5 \%$. The most common complications are usually infection of the wound site or pain from too rapid injection of Ethiodol. Rarely, extravasation of Ethiodol into the soft tissues of the feet may cause pain and discomfort for protracted periods (months). ${ }^{9}$

In a study of 42 patients with a lymphatic leak, Cope et al demonstrated a cure with thoracic duct embolization and needle disruption in $74 \%$ of the patients with no morbidity with a follow-up period of 3 months. ${ }^{10}$ In 7 of the 42 patients (17\%), access into the thoracic duct could not be obtained, and these patients went on to surgical thoracic duct ligation. In the majority of cases, inability to access the thoracic 
duct was due to absence of a suitable access target, such as a cisterna chyli. This may pose a problem because the cisterna chyli is visualized on only 35 to $50 \%$ of lymphangiographic studies. ${ }^{11}$ In these cases, it may be possible to access the lymphatic system via large retroperitoneal trunks. In a more recent study, Binkert et al demonstrated a $100 \%$ success rate without morbidity in the treatment of thoracic duct injury by percutaneous embolization with coils and glue combined with needle disruption of the cisterna chyli. However, the sample size was small (8). ${ }^{12}$

\section{CONCLUSION}

As more studies are conducted in this field, it has become evident that a lymphangiogram with needle disruption has found a niche in the treatment of lymphatic leaks. It is a safe and viable technique in patients who are poor surgical candidates or whose leaks are not responding to conservative measures.

\section{REFERENCES}

1. Barnacle AM, Kleidon TM. Lymphatic leak complicating central venous catheter insertion. Cardiovasc Intervent Radiol 2005;28:839-840

2. Porziella V, Cesario A, Margaritora S, Granone P. Role of pre-operative lymphangiogram and lympangioscintigraphy in the surgical management of spontaneous chylothorax. Eur J Cardiothorac Surg 2006;30:813
3. Merrigan BA, Winter DC, O'Sullivan GC. Chylothorax. Br J Surg 1997;84:15-20

4. Leibovitch I, Mor Y, Golomb J, Ramon J. The diagnosis and management of postoperative chylous ascites. J Urol 2002; 167:449-457

5. Yamagami $\mathrm{T}$, Masunami $\mathrm{T}$, Kato $\mathrm{T}$, et al. Spontaneous healing of chyle leakage after lymphangiography. Br J Radiol 2005; 78:854-857

6. Cope C. Diagnosis and treatment of postoperative chyle leakage via percutaneous transabdominal catheterization of the cisterna chyli: a preliminary study. J Vasc Interv Radiol 1998;9:727-734

7. Dugue L, Sauvanet A, Farges O, Goharin A, Le Mee J, Belghiti J. Output of chyle as an indicator of treatment for chylothorax complicating oesophagectomy. Br J Surg 1998; 85:1147-1149

8. Cerfolio RJ, Allen MS, Deschamps C, Trastek VF, Pairolero PC. Postoperative chylothorax. J Thorac Cardiovasc Surg 1996;112:1361-1365

9. Rubin BE. Extravasation of ethiodol into deep tissues of the foot: a complication of lymphangiography. AJR Am J Roentgenol 1978;131:342-343

10. Cope C, Kaiser LR. Management of unremitting chylothorax by percutaneous embolization and blockage of retroperitoneal lymphatic vessels in 42 patients. J Vasc Interv Radiol 2002; 13:1139-1148

11. Pinto PS, Sirlin CB, Andrade-Barreto OA, Brown MA, Mindelzun RE, Mattrey RF. Cisterna chyli at routine abdominal MR imaging: a normal anatomic structure in the retrocrural space. Radiographics 2004;24:809-817

12. Binkert CA, Yucel KE, Davison BD, Sugarbaker DJ, Baum RA. Percutaneous treatment of high output chylothorax with embolization or needle disruption technique. J Vasc Interv Radiol 2005;16:1257-1262 